sence reaches the ear; a cavity connected with the surface by continuous consolidation may, and probably will, give evidence of itself by a peculiar modification of the breath sounds, if not of the voice sounds also, that indicate consolidation. Over cavities of less than a certain size you must not expect to find the true "amphoric" breathing, the sound as of the breath passing over the mouth of a vase, any more than "metallic tinkling " or "echoing cough"; but even over small cavities the breath sound has a definite and readily recognizable peculiarity, which entitles it to the name of "cavernous breathing." Now cavernous breathing is sometimes difficult to make sure of unless you are provided with a definite criterion by which to distinguish it from the tubular breathing of which it is really a modification. Such a criterion is not to be found in the inspiratory part, which differs little, if at all, from that of tubular breathing, but it is furnished by the expiratory part, and the distinction involves a question of pitch. In tubular breathing due to consolidation the expiratory sound is invariably of a slightly lower pitch than the inspiratory; while over a cavity the difference is reversed. Curiously the distinction of pitch does not strike every one alike. The expiratory sound of cavernous, as of tubular, breathing is a "noige" made up of vibrations of very various lengths, and some ears appear to be more sensitive to the higher and some to the lower vibrations among them. The former convey the general impression of a relatively high pitched, the latter that of a relatively low-pitched sound. Some authors, you will accordingly find, represent comparative lowness of pitch, instead of comparative acuteness, as characteristic of the expiratory part of cavernous breathing. I have several times brought the question to a practical test in $\mathrm{my}$ classes in the wards, and every time with the result that to the majority, as to myself, the cavernous expiratory gound appears to have a higher pitch than the inspiratory. Whichever way the difference impresses your individual ear. you will find it constant for nearly all cavities. In most persons, sounds of the character I have described as cavernous may be heard if the stethoscope be applied over the trachea. This suggests a caution in interpreting dry cavernous breathing heard either above the clavicle or over the back near the windpipe. In either case it may chance to be only tracheal breathing conveyed through consolidated lung. Error is best avoided by comparing the supposed cavernous breathing with the actual tracheal sound.

Pectoriloquy is of less value as a sign of cavitation than cavernous breathing. It is not always heard over small cavities, and it is occasionally heard when no cavity exists. I will devote a few words to the definition of this term, as it is not infrequently quite misused. Normal vocal resonance, as I have already said, is a . confused "buzz" conveying a general impression of the vocal sounds uttered, but no distinct idea of the articulation. Vocal resonance may be augmented or may even: become bronchophonic without becoming distinctly articulate. In this case it should not be called pectoriloquy; the term is intended to imply the distinct conveyance of articulate speech to the listener's ear. Pectoriloquial conduction is more easily. detected when the whispered is substituted for the spoken voice, but the mere conduction of the whispered sound, as long as it is in the "buzz" form, does not constitute pectoriloquy, or even fürnish evidence of disease. It often takes place through healthy lung. The sign to which many years ago I gave the name of "post-vocal whiff," I mean the faint short expiratory puff which is sometimes heard when articulation has ceased and the lips of the glottis have flown open to give exit to the compressed air in the trachea, is of no value as a diagnostic mark of a cavity. It sometimes occurs over a cavity, but quite as often over merely consolidated lung in the apex.

Up to this point. I have had young subjects, the most usual victims of tubercle, mainly in mind. But tuberculosis of the lung may break out at any age. In the elderly its first onset has frequently to be recognized by a somewhat different set of signs. After a certain age the disease is more commonly the sequel of a chronic non-tuberculous bronchitis. The bronchitis may have existed on and off for many years, appearing under the guise of a "winter cough" and disappearing in the spring, and the early signs of tubercle have to be recognized among those of a generalized bronchitis which may have become familiar by repetition. The earliest sign to be discerned is usually neither dullness nor harsh breathing, nor alteration of voice sound, but a local change in the character of the bronchitic rhonchus. The pitch of a bronchitic rhonchus in the upper part of the chest, whether inspiratory or expiratory, does not usually vary much throughout the individual inspiration or expiration, whichever it be. If the inspiratory rhonchus at one or other apex become broken in the middle, the latter part becoming markedly high pitched, so as to give to the ear the impression of a creak, it should arrest attention. "Creaking" rhonchus (the creak is more often inspiratory than expiratory) in the adult should always convey a strong suspicion of tuberele. If, in addition, the inspiration should bring out sharp clicks, or even small râles of a higher pitch than is found over the rest of the lung, the proof is almost formal. I am speaking, you will remember, of the adult, and practically of the elderly, subject. In children these signs have no such significance ; they are quite common in ordinary bronchopneumonia. In the elderly I have never, to my recollection, heard them at the apex except in lungs which were subsequently proved to be tuberculous, either by bacteriological examination or by the subsequent progress of the case.

\section{THE EARLY DIAGNOSIS OF TUBERCULOSIS OF THE LUNGS.}

BY C. G. H. BAUMLER, M.D., F.R.C.P.LoND., Professor of Clinical Medicine in the University of Freiburg-i.-Br. formerly Assistant-Physician to the German Hospital, Dalston and the Hospital for Diseases of the Chest, Victoria Park. London.

WITH the progress of our knowledge of pulmonary tuberculosis the desirability or rather the necessity of recognizing in patients the very first changes has greatly increased, more especially since a more hopeful view as regards the curability of the disease has replaced the former despondency. We now know, to a certain extent at least, the way and the means by which the organism gets rid of the changes which the tubercle bacillus brings about, at first quite locally, in the tissues. Local inflammatory reaction and the formation of connective and, further on, of fibrous tissue, which means the replacing the diseased lung-substance by a cicatrix, are the prominent features of this healing process in which, by reactive functions of various kinds, the whole organism is more or less involved. In many cases the original strength of the body is sufficient to fight the battle even amidst unfavourable surroundings, but in the majority of patients in whom already a certain dégree of debility had been the cause that the disease could have taken root, or could have been developed, assistance by external ineans gives the only chance of recovery. Brehmer and Dettweiler, Spengler, Sir Hermann Weber, and other pioneers on the line of the climatic treatment of tuberculosis of the lungs have shown the way by which this external support can best be offered to the patient, and by which unexpected results have already been obtained in ever increasing instances.

But this, no less than other measures-in fact, the whole modern crusade against tuberculosis-can, apart from prophylaxis, only be successful, if patients get under appropriate treatment as early as possible, when only few and small foci of disease are present, and as long as the destruction and replacement of them by fibrous tissue does not materially interfere with the normal aetion of the lungs or unfavourably react on the whole organism.

If by the progress in our knowledge of the disease and of the possibility to battle against it, the power of the phyisician has been greatly extended, his responsibility also has enormously increased, involving, as it does, the paramount duty, if possible, to recognize the very beginnings of the disease. These being at first purely local, and remaining so for various lengths of time, to make out the signs of these local changes will be of special importance.

In the majority of cases a somewhat persisting cough is the only symptom which induces a patient, or more often compels his friends, to seek medical advice. Very commonly, however, other symptoms, subjective as well as objective, are already present, which, in connexion with cough or even without it, may raige a suspicion that tubercles may have formed in the lungs. Foremost among these are febrile symptoms, lassitude, pallor, slight shiverings, night sweats, loss of appetite and weight.

Whenever such symptoms are complained of or suspected in a patient, they have to be carefully investigated, being of great value for the diagnosis, and very often even more still for the opinion which we shall form of the prospects for recovery and of the further progress of the case. Increased attention should be paid to these general symptoms, 
whenever the examination of the lungs gives at first no positive results.

Of first importance in this respect is a careful observation of the body heat, the temperature being measured for a number of days three times a day, noticing also the effect of rest and exercise on its range. If the temperature be raised ever so little above the normal standard, the observations must be continued for a certain period and at the same time, the diet being carefully regulated, the body weight ought to be taken at regular intervals.

A continuous, even slight, elevation of temperature, or a loss of weight, if not explained by other discoverable disease, is extremely suspicious of pulmonary tuberculnsis, which then, sooner or later, will become apparent by distinctive local signs. In childhood, and at the age of puberty, tuberculosis of the lymphatic glands in the chest and abdomen may be the cause of such a general decline, to which, by-andbye, lung symptoms are superadded. As in cases of this kind the spleen generally is somewhat enlarged, a suspicion may arise that the case might be one of typhoid fever of a lingering type. But the spleen hardly ever reaches the size it attains in typhoid fever, and typical rose spots never appear, whereas miliaria crystallina (sudamina), which so commonly appears in typhoid fever after the midale of the third week, may be present in either case when profuse perspirations occur. To distinguish typhoid fever from such a case of glandular, or, maybe, of latent pulmonary tuberculosis, the examination of the blood for the typhoid bacillus, the counting of the leucocytes, which in typhoid fever are diminished in number ("leucopenia"), and the Widal test ought to be employed, and will generally decide the diagnosis. Diazo-reaction of the urine may be, for a time, present in either case.

The local signs of pulmonary tuberculosis vary according to the kind of change which the disease brings about. Alveolar and lobular infiltration and bronchial catarrh being the coarse anatomical basis of the disease, diminished or altered resonance on percussion, and altered breath sounds on auscultation will be the chief signs that have to be looked for in the beginning, more especially over the apices of the lungs, which in the overwhelming majority of cases are the first parts to be affected. A careful comparison of the two sides and of corresponding points will lead to the discovery of very slight changes. Regard, however, must be had to the differences which are normally present, in somewhat varying degrees, between corresponding places of the two sides. Generally the percussion note is slightly louder on the right than on the left side, above as well as below the clavicle, unless the muscles are unequally developed on the two sides, those on the right side, at least in men, frequently showing a somewhat better nutrition.

The places which ought to be most carefully compared are the supraclavicular region and the lateral portions of the first and second intercostal spaces, a diminished percussion note at the apex generally showing itself not only above, but generally, in a slighter degree, also below the acromial portion of the clavicle. A marked dullness of the percussion sound in these parts of course already signifies a somewhat extensive infiltration, involving a good many lobuli, and distinct augcultatory signs, to be discussed hereafter, will then also not be wanting.

At the back, in the supraspinal region, the percussion note may be in such a case the same as on the other side, if the diminution of air in the apex of the lung by infiltration is confined to its anterior parts. The percussion sound may then even be somewhat hyper-resonant and slightly tympanitic in consequence of relaxation of the otherwise normal lung tissue.

A marked difference on percussion may, however, be present without any lung disease in the apices, when the two sides of the chest are nut perfectly symmetrical. Slight asymmetry is very common, and is generally due to rickets having been passed through in infancy.

There need, in such a case at first sight, not be any vers marked irregularity of shape, especially of the front aspect of the chest, but a more careful comparison of the two supraclavicular regions, and the inspection of the back and the examination of the vertebral column, will rbveal more or less marked signs of scoliosis and its influence on the position of the ribs and the shape of the chest. The vertebral column, as judged by the spinous processes, may appear quite straight, although the upper ribs on one side are more curved and on the other somewhat flattened, which may be due to slight rotation of a number of vertebrae or the normal kyphosis of $/$ the dorsal portion is more or less abolished with secondery diminution of the cervioal and lumbar lordosis, not to speak of pronounced lateral curvature. All these deviations reaet on the position and shape of the ribs, and thereby influence the shape of various parts of the chest.

Even very slight deviation of the vertebral column from its normal shape causes a difference in the height of the two supraclavicular regions and in the sagittal diameter of the upper part of the two sides of the chest, the supraspinal region on one side being at the same time more rounded than on the other. Percussion on this somewhat more convex side will produce a less resonant note than on the more flattened one. In the same manner does the altered shape of the supraclavicular space influence the percussion sound. Generally the supraclavicular space appears larger and higher on the side on which the supraspinal region is more convex. Thus the percussion sound may differ both in front and at the back in an opposite sense without there existing any disease of the lungs at all, and the loudness of the respiratory murmur is likewise thereby affected.

Even a slight deviation of the vertebral column, especially an abnormal lordosis of its dorsal portion, which may not react at all on the front aspect of the ehest, but only produces a certain widening of the middle intercostal spaces, may also materially influence the position of the heart, the shape and size of the heart's dullness on percussion, and the place and quality of the apex beat. The sternovertebral diameter of the chest being diminished, the heart generally slides somewhat to the left, and is less covered by the anterior borders of the lunge. In consequence thereof the heart's impulse may be somewhat displaced to the left, and the heart's dullness increased. If the intercostal spaces are wide, the apex beat may appear abnormally extended and very strong, although the heart itself is quite normal. Grave errors of diagnosis, as regards both the lungs and the heart, may result from not paying due attention to these things.

As not only the air capacity of the alveolar tissue of the lungs, but its tension also is affected by tuberculous infiltration, the percussion sound may, in places and at times, become hyper-resonant, and of a tympanitic quality. This, however, is much more frequently the case when infiltration of a more extensive area occurs in the lower regions of the lungs, especially below the scapula, or in front and at the sides in the lower intercostal spaces. At the apices a well-marked tympanitic resonance generally signifies not the commencement of an infiltration but a more advanced stage, the lung tissue being, in fact, quite deprived of air. Through such a homogeneous airless tissue the vibrations of percussion reach the bronchial tubes and cause the air column contained in them to vibrate, whereby tympanitic resonance with Wintrich's sign-namely, change of pitch on opening and closing the mouth or the nostrils-is produced. The cracked-pot sound may also appear under such circumstances on strong percussion. The same physical causes obtaining when cavities have formed within an infiltrated apex of a lung, either by softening and ulceration or by dilatation of affected bronchial tubes, these signs are generally, but, I venture to say, very often wrongly, attributed to cavities. They may be, and frequently are, due to cavities, but solid infiltration down to the larger bronchial tubes, as in acute pneumonia of an upper lobe, causes exactly the same signs on percussion. In order to be justified to refer them to cavities, auscultatory signs must be called in aid. But signs such as occur over cavities never belong to the first but to the more advanced stages of pulmonary tuberculosis, and need, therefore, not be gone into further.

As already mentioned, tuberculosis of the lungs does not invariably commence in the apices; and what one observes sometimes clinically favours the view first brought forward, on the strength of pathological investigation, by BirchHirschfeld, ${ }^{1}$ that tuberculosis, as a rule, commences with a local affection of the mucous membrane in one of the larger bronchi of the upper lobe. In such a case infiltration of the surrounding lung tissue will be caused by the tuberculous process spreading beyond the walls of that bronchus and also by inflammatory changes which may be started in the area of lung tissue to which the affected bronchus leads. By a local swelling and ulceration of the mucous membrane of a bronchus the passing in of the air in inspiration, but more especially its passing out in expiration, will be interfered with. Collapse (atelectasis) of airvesicles and lobules, as well as emphysematous inflation of parts of this area, may be the consequence. As soon as ulceration takes place in the bronchus, infection, not only by the tubercle bacillus but also by. other bacteria of the septic class, may occur both by spreading along the lymphatic channels, as by particles of pus and cast-off tissue being sucked in with deep inspirations, 
such as will follow a fit of coughing, or they may be pushed forward by. the increased intrabronchial pressure in the act of coughing.

Auscultatory signs, of which we shall have to speak presently, and the occurrence of signs of a lobular pneumonia, first showing itself in the second or third intercostal space towards the axilla, may possibly be due to such a primary bronchial localization of the disease. In two cases that have come under my observation the first signs of lung disease in well-built and well-nourished young girls appeared quite acutely in the form of a lobular pneumonia in the third left intercostal space, spreading gradually towards the axillary region, the apex being apparently unaffected in one case, whilst in the other one slight signs of apical infiltration were present. In another case I have seen the first distinctive signs of a tuberculous infiltration to show themselves below the right scupula.

Generally in these, as in fact in a great number of cases of the ordinary apical commencement of the disease, it is not percussion, but auscultation, which yields the first suspicious or well-marked signs. In cases such as those alluded to crepitation or a coarse râle caused by the bursting of one or a few bubbles of mucus, taking place especially. at the first inspiration after a cough, are sometimes the very first local signs. As a locality where râles ought to be sought for, the medial end of the scapular ridge, in fact the upper part of the whole interscapular space, may be pointed out. Not infrequently this locality, and more often on the right side is the only one where râles can be found on deep inspiration, and more especially after having induced the patient to cough. With such râles-a kind of crackling-however, an auscultatory sign very similar in Nature, must not be confounded-namely, the bubbling or crackling noise which is caused by swallowing frothy saliva, and which may be accidentally heard on auscultation of the interscapular region. As it only appears in the act of swallowing, and not with respiration, an error can easily be avoided.

Persistent and strictly localized râles of various kinds, more especially in the upper parts of the lungs, are among the most important signs of commencing as well as of already advanced tuberculous disease of the lungs.

Not so certain in their significance, but more common and always suspicious, are deviations of the respiratory murmur from its normal character in the various parts of the chest, but more especially over the apices. The respiratory murmur varying considerably in different individuals, it is chiefly a marked difference between corresponding parts of the chest which is of diagnostic value. The normal vesicular murmur being generally a little louder in the right than in the left supraclavicular and infraclavicular region, and an expiratory murmur over the right apex being audible in many cases of undoubted soundness of the lung the reverse condition must be considered suspicious. A marked diminution of the inspiratory breath sound on one side, and more especially so on the right side, if persistent on repeated examination, must rouse a suspicion that this part of the lung contains less air. This may mean that its inspiratory inflation is somewhat impeded by narrowing of some bronchioli leading to it, or that a number of lobuli are imperviously infiltrated.

A prolonged expiratory murmur, especially on the left side, may be caused by catarrhal or other narrowing of small bronchial tubes, or it may be also, by changes in the lung altering the influence which the vesicular lung tissue in its normal condition has in modifying or extinguishing the murmur produced by the respiratory movement of the air at the glottis and in the larger bronchi. If this influence is entirely abolished by complete infiltration of an area of some extent, then the breath sounds, as generated at the glottis and at the divisions of the bronchial tubes, are conducted to the surface almost unmodified, so that tracheal or bronchial breathing will be the result.

Very frequently the breath sounds appear coarser than normally, and evidently not so uniform as regards the succession of vibrations causing them. They lack more or less the characteristic vesicular (breecy, schlürfende) quality of the normal breath sound. If weaker, they may resemble the murmuring noise which is heard on auscultating a large muscle in the act of contraction; if stronger, they are of a harsher quality than normal breath sounds, though quite different from and less sharp than, the harsh vesicular breath sound heard in chronic dry bronchitis of elderly people, as also sometimes in the very first stage of acute pulmonary infiltration preceding, and in many placess intermixed with, fine erepitation.
There are, however, such manifold graduations from the normal vesicular breathing of young and quite healthy people to this very coarse respiratory murmur of an acute or chronic bronchial catarrh, and to the bronchial breathing of infiltration, that a description of all the forms becomes very difficult. It is therefore not to be wondered at that a term like indeterminable (umbestimmtes Atmen) could have been introduced into auscultatory terminology. No distinct changes in the lungs can be looked at as the equivalent pathological substratum of every one of the various deviations from the normal breath sounds. A reduction of the air contained in a portion of lung or an altered capacity of the air vesicles, and catarrhal or other narrowing of bronchial tubes are their chief causes.

A difficulty which may render auscultation quite fruitless sometimes arises in nervous persons, especially women. When asked to draw their breath they produce forcible movements of the chest, closing, however, at the same time, the glottis, so that actually no air can enter the lungs, and no breath sounds, of course, can be heard. In such a case of disturbance of the normal co-ordination of the various muscles acting together in inspiration one may, by patient instruction, gradually overcome the nervousness and get the patient to breathe properly. If this fails, in some rare instances together with the goodwill of the patient, it is well to make him cough. The forcible expiration thereby caused compels him afterwards involuntarily to draw air into his lungs:

The reverse--namely, very much louder and harsher-breath sounds than should be expected from the other circumstances of the case-is sometimes observed when nervous patients, by narrowing the glottis, instead of widening it. on inspiration, or by contracting their isthmus faucium, produce a noisy stridor in the throat, which, not only by the loudness with which it is transmitted through the air, interferes with auscultation, but overshadows also the breath sounds in euch a manner that their character becomes more or less bronchial. The same may occur in patients in whom a thyroid swelling or other diseases, including paralysis of one or both vocal chords, cause a narrowing of the upper air passages.

The limits of a focus of tribcrculous disease in the lung are sometimes marked by a zone of very sharp, distinctly vesicular breathing, which not infrequently shows interruptions-"cogged breathing," "saccadiertes Atmen"--due probably to limited narrowing of bronchial tubes, allowing the air in inspiration to pass on towards the vesicles only when the suction exercised by the widening of the chest acquires a certain strength.

The velocity with which the air enters into those alveoli which are quite normal and into adjoining ones which are partly or wholly infiltrated, and with which it passes through normal or narrowed bronchioli, must, of course, vary considerably, and it is chiefly this velocity of the air current on which, to a.great extent at least, the character of the breath sounds depends.

of very great importance for diagnosis may be very fine crepitation, of greater importance still when heard only for a limited period on thé same spot, then disappearing or being replaced by coarser râles. This would indicate that the changes causing the crepitation have gone on developing into more complete infiltration or even into softening. Such a change would indicate advancing disease. If, however, very fine crepitation be heard on one and the same spot for weeks or even months, it is evidently due to chronic changes, and may be a sign of very slight importance or of none at all, as an indication of tuberculous changes. It may simply be due to collapse of a number of air vesicles or lobuli, the inflation of the alveoli by deeper inspiration causing this fine and equable crackling noise. In support of this view it may be mentioned that such crepitation is commonly heard on the borders of the lungs, especially the lower ones, as also along the pericardium, more especially on the left side, in some rare instances over a clavicle, when old cicatricial changes are present in the apex.

Along the border of the left lung adjoining the heart fine inspiratory crepitation is often heard in cases of heart disease, in which no question. at all can arise as to tuberculosis of the lungs. Over and over again it has occurred to me to see patients which had been considered to be cases of lung disease, and had been given creosote and other drugs in vogue at the time, in whom, however, a careful examination and prolonged observation showed that actually no lung disease was present, but that occasional slight haemoptysis, which had first caused a suspicion of tuberculosis of the lungs, was due to valvular disease of the heart. It had been 
chiefly this haemoptysis which had led to an erroneous interpretation of crepitant râles, heard along the border of the left lung adjoining the heart. Haemoptysis frequently occurring in cases of mitral stenosis, quite apart from embolism and infarctus of the lung, and being simply caused by congestion due to failure of the left ventricle, it is very natural that, when cough also is complained of and crepitant râles are heard in the neighbourhood of the apex of the lung, tuberculous changes are thought of, especially when the valvular disease is overlooked. This may easily occur, for of all valvular diseases of the heart mitral stenosis has, as is well known, the most varying auscultatory signs - a murmur, in fact, at times being quite absent-whereas at the next examination, or if the heart's action gets accelerated by exercise or nervous influence, a loud characteristic presystolic murmur may be heard. If the heart is not very carefully examined and only whilst the patient is standing, the very loud first sound at the apex, which is generally present in these cases, may be taken for an increased second pulmonary sound and explained by an assumed narrowing of the pulmonary circulation in consequence of chronic changes in the lungs due to tuberculosis. A comparison of the heart sounds with the pulsation of the carotids and a careful examination of the heart, the patient being in a recumbent position, as well as of the lungs, will easily correct any erroneous presumption which may at first have been formed.

In such a case the temperature, unless raised by other causes, is of course quite normal, and in the sputum, by repeated and careful examination, tubercle bacilli are not found, and no distinct signs of a really tuberculous disease in the lungs can be discovered.

As regards the discovery of tubercle bacilli in the sputum, it need hardly be mentioned that their absence on repeated careful examination in a given case is no proof that a disease of the lungs, which, by its clinical symptoms, might be taken to be due to tuberculosis, is really not due to it. Their absence in the sputum only proves that at the time no destruction of a tuberculous focus is taking place. They may appear with the further progress of the case. Of greatest importance, on the contrary, for the diagnosis in a given case may prove their discovery, when all the other symptoms are very slight and when no focal disease can be made out in the lungs. In such a case there must be a focus somewhere, but so much surrounded by normal.lung tissue, that neither percussion nor auscultation yield any positive sign of its existence. There may be very little cough and only occasionally a little expectoration of purulent sputum. This may come from a central, perhaps original]y bronchial, focus in the lung, or from perforation of a softened tuberculous bronchial gland into a bronchus.

Frequently-repeated careful examination of the sputum is particularly desirable in cases in which the good general health and aspect of a patient who is troubled with cough seems to negative the idea that a tuherculous disease might be its cause. Patients of this kind are generally beyond middle age, rather stout than the reverse, and at first sight look more like being, affected wilh emphysema and chronic bronchitis, or with heart disease. But the examination of the lungs reveals considerable changes at one or even both apices. There is a history of haemoptysis some time in early life, after which, however, perfect general health had soon been established and maintained. There had at first been but slight cough, which only lately somewhat increased. The changes found, which chiefly consist in induration, including sometimes small cavities, are the remnants of an old attack of tuberculous disease from which the patient had recovered, but where quite recently fresh changes were taking place. These are the cases in which haemoptysis occurs, at times when by catarrhal or other causes congestion of the bronchial and pulmonary blood vessels is induced

Should there exist an aneurysmatic dilatation of a branch of the pulmonary artery lying in the wall of a small cavity, profuse, even fatal, haemorrhages may occur in a patient who, as regards his general condition, does not in the least rouse a suspicion that he might be affected with tuberculosis. Considerable haemoptysis in cases of this kind sometimes also ushers in an acute attack of multiple broncho-pneumonia, caused by the aspiration of the bacillary contents of a cavity. Several cases in which before the fatal illness the patients had appeared perfectly healthy, but were carried off in a galloping consumption within a few weeks, have come under my own observation. ${ }^{2}$ The initial haemoptysis may be a symptom of the commencing inflammation, sometimes, however, when brought on by violent exercise, its very cause, the dyspnoea which accompanies profuse haemorrhage from the lungs leading to aspiration of blood and of the contents of a cavity into all parts of the lung.

Haemoptysis, with very trifling quantities of blood, but frequently repeated, is sometimes the first and only symptom that raises a suspicion of tuberculosis of the lungs. In such a case the percussion may give normal results, but the breath sounds are harsher than normally, and there may be slight catarrhal rhonchi at the base of both lungs. The scanty sputum contains no tubercle bacilli. There is no elevation of temperature and the general health is unimpaired. All that can be found on repeated careful examination is hypertrophy of the tonsils and chronic pharyngeal catarrh. This is really the cause of the haemoptysis. I have repeatedly seen cases of this kind in which for years afterwards, no signs of tuberculosis were developed.

Another throat affection, also, may sometimes be a source of erroneous diagnosis or of a suspicion, at least, that tuberculous lung disease might be present. This is enlargement of the thyroid gland or of one of its lobes pressing on the trachea, but not yet causing any noticeable impediment to respiration, unless a respiratory effort be made by heavy work or violent exercise. In such a case there arises, sooner or later, a tendency to local congestion and catarrh in that part of the mucous membrane where the trachea is slightly pressed on by the thyroid gland. This causes cough and generally a scanty and purely mucous expectoration. If from various causes this catarrh attains a greater intensity, the expectoration may even be tinged with a little blood. On auscultation rather rough breath sounds are heard all over the lungs, more especially also at their bases, and a prolonged expiratory murmur is heard everywhere. Where this condition of things had existed for some time there is generally some emphysematous enlargement of the lungs. In cases of this kind all the other symptoms which may be of importance for diagnosis have to be carefully gone into and.weighed before a decided opinion can be given.

Recurrent slight or somewhat profuse haemoptysis may also be caused by an aneurysm of the aorta pressing on the trachea or a large bronchus. All other symptoms or signs of an aneurysm may be wanting, but its existence will be disclosed by tracheoscopy.

When +ver all diagnostic methods spoken of give no decisive result, there remains the tuberculin test as a most valuable means for settling the question. This is best employed according to the method observed at the Institute for Infectious Diseases at Berlin. ${ }^{3}$

The normal course of the temperature of a patient having been carefully established by measuring it for two days every two or three hours, a subcutaneous injection of $\mathrm{mg}$. of the old tuberculin is made under the skin of the back in the evening between 6 and $80^{\prime}$ clock, as the reactive rise of temperature generally begins after twelve hours. For children the dose must be smaller. After one or two days $5 \mathrm{mg}$. and, if necessary, after another one or two days, so mg. are injected. A rise of temperature of at least $0.5^{\circ} \mathrm{C} . \mathrm{x}^{\circ} \mathrm{F}$.) is considered a positive reaction. The solution of tuberculin should always be freshly prepared. Patients that are feverish should not be injected.

The serum test of Arloing and Courmont, which is based on the agglutination of a pure culture of tubercle bacilli being produced by blood serum from patients affected with tuberculosis is, even with the improvements introduced by $R$. Koch, ${ }^{4}$ no method suitable for every day practice.

Many things, therefore, as will have been seen from the foregoing remarks, must be taken into consideration and carefully examined before we can arrive at a sure diagnosis in cases of commencing tuberculous lung disease. Diagnosis gets more difficult when no very positive lung signs can be discovered and when there is no expectoration in which tubercle bacilli can be looked for. In such a case a most searching examination must be made over and over again to get at a decision, upon which the gravest issues may depend. But if, on the one side, it is a matter of greatest responsibility not to have diagnosed really existing tuberculous disease, and thereby to have allowed the most favourable time for recovery to pass by unused, it is, on the other no less a fault to take away any one, especially youths, from their work and, may be, from their family, and put them on the path of health-seeking invalids, if such a course is not really necessary. But what course had best be taken when the first signs of a pulmonary tuberculosis have been actually made out, whether sanatorium or home treatment, whether at a high altitude or on the sea-level, or at an intermediate station - all these and other questions have to be carefully considered and must be decided according to all the circumstances of each individual case. 
REFERENCBS.

1 Deut. Arch. f. klin. Med, Bd. Ixiv, 1899, p. $5^{8}$. 2 Uber eine besondere durch Aspiration von Caverneneninhali hervorgerufene Form acute Bronchopneumonie bei Lungentuberculose, Deut. med. Woch. 1892 ${ }^{3}$ Max Beck, Uber die diagnostische Bedeutung des Koch'schen Tuberculins, Deut. med. Woch., 1899, No. 9. Deut. med. Woch., 1901, No. 48.

\section{ABSTRACTS OF}

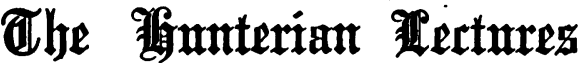

\author{
ON THE
}

\section{PATHOLOGY AND SURGERY OF CERTAIN DISEASES OF THE PANCREAS.}

Delivered before the Royal College of Surgeons of England. BY A. W. MAYO ROBSON, F.R.C.S., Hunterian Professor.

\section{LECTURE III.-CYSTS, INJURY, NEOPLASMS.} Calculus, Neoplasm, Pancreatic Cysts.

THE surgery of cystic disease of the pancreas is much in advance of pancreatic surgery as a whole. All authorities are agreed as to the wisdom of simple drainage, which is usually sufficient to bring about relief or cure. Experience has shown that the patient's interest is best considered by a limitation of the incision to a size sufficient to empty and drain it, and not sufficiently iarge to satisfy pathological requirements. Hence it is highly probably that many reported cases of operations for pancreatic cyst have been for cysts of other origin, and it is an undoubted fact that quite a number of cysts originating from the neighbourhood of the pancreas are only pseudo-cysts, as in two of my own, where in one a collection of blond and serum filled the lesser peritoneal sac as a result of injury; and in another a pseudo-cyst formed rapid] y around a necrotic pancreatitis, and from the cavity a slough of the pancreas was removed.

The symptoms produced by a pancreatic cyst vary according to the size and seat of the tumour. They are at first dependent on the disease leading to the cystic formation, and later to the pressure exercised by the tumour on neighbouring viscera; so that we find digestive disturbance, with loss of flesh and epigastric uneasiness, or some pain, quite early, these being followed in certain cases by very intense pain occurring in paroxysms, by vomiting, constipation, jaundice, and wasting, and later by the presence of a tumour. Fatty stools, azotorrhoea, bulky pale motinns, and glycosuria will be present or absent, according to the amount of degeneration present in the gland. In all the cases I have observed recently there has been a well-marked pancreatic reaction in the urine. indicating catarrh of the pancreatic ducts or interstitial inflammation; and if this test holds good for cystic disease of the pancreas generally, as I believe it does, it will, I think, form an important diagnostic sign in any case of tumour suspected to be pancreatic.

The most frequent cause of cyst development is chronic interstitial pancreatitis, in which compression and constriction of the ducts result from a new formation of connective tissue with consequent stagnation of secretion. The Roentgen rays may also form a uselul help in diagnosis in certain cases, as they will establish the presence or absence of pancreatic calculi, which are quite opaque to the $x$ rays.

It is to be borne in mind that there have been cases of pancreatic cyst presenting very few symptoms except the presence of a tumour which has been under observation for a long time and has needed no active treatment. but these cases are exceptional.

Treatment.

Occasionally excision of the cyst may be performed, as in a case of my own, in a woman aged 38 , where the tumour returned after drainage, and where the patient made a complete recovery after the second operation; but the greater difficulty in performing excision, the impracticability of doing it always and the greater mortality attending it, as compared with the old operation of incision and drainage, renders it quite clear that the lesser operation should always be tried first unless the circumstances be very exceptional. Aspiration and other forms of tapping are antiquated and ineffective methods attended with more danger than the operation of incision and drainage, and therefore not to be recommended even for diagnostic purposes; this also applies though in a lesser degree to operation $a$ deau temps which is now rarely performed.
As to the situation for drainage, that must entirely depend on circumstances; the cyst will usually be attacked from the front, where it is manifestly nearest the surface. Occasionally it may be drained from the loin as in one of my cases.

Fistula does not as a rule follow the drainage of pancreatic cysts, but in some cases a small fistula maypersiat, and 1 have known a fistula to go on for years without hurt to the patient, and with very little discomfort.

In the cases I have operated on a cyst was enucleated on one occasion, recovery following ; drainage was carried out in 9 other cases of true cyst, and was-followed by recovery in 8 . whereas of 2 pseudo.cysts, one due to traumatic hremorrhagic pancreatitis and the other to necrotic pancreatitis, I recovered. These I shall hope to report more fully before one of the London societies, as the time will not permit me to dwell longer on them now.

An extensive search through literature, in which I have been a ssisted by Mr. A. Clarke, who has verified the references for me, reveals the following facts :

r6o cases of operation have been recorded.

In 140 recovery took place, or is presumed to have taken place. In 4 cases the ultimate issue was doubtful.

In 8 out of the $x 40$ of reported recovery after operation the patient died subsequently : I from diabetes, four months later ; I from tuberculosis, three months later; I from haemorrhage, a year and a-half later I from concomitant peritonitis, 7 weeks later; I from epidemic fever, a few weeks later; 3 from causes not stated, a few weeks later. Death is recorded as the result of operation in 20 cases. In 5 of these the canse and time after operation are not given. One patient died in collapse; c I eighteen days after operation (cause not stated); 2 from shock; I from gangrene of the pancreas; 8 from peritonitis. Of these 8 dying from peritonitis 1 died at interval not stated, I after ninety-six hours; I after six days ; $x$ after an exploratory incision ; 2 after two days : I on eighth day ; 1 on second day.

In 38 incision and drainage were performed, with 6 deaths, equal to a mortality of $1 \times .6$ per cent.

In 5 excision was performed, with 3 deaths, equal to a mortality of 20 per cent.

In 7 partial excision was done with 3 death, equal to a mortality of I 4.3 per cent.

Although larger numbers have been reported by others the above figures are as nearly correct as appears to be possible. for on verifying the records sometimes the same case had been reported twice, in others wrong dates had been given, and in a few the details were so margre that even the nature of the operation was not given. The evidence is clearly in favour of drainage, but the mortality should certainly be reduced by one-half.

As to the frequency of cysts of the pancreas, Dr. Hale White has secorded that in nearly 6,000 necropsies at Gny's Hospital (1883 to 1894) pancreatic cysts were only found in 4 cases, and one of these was a hydatid cyst.

Injuries of the Pancreas.

It is quite clear that injury to the pancreas is not necessarily fatal, as shown by the development of cysts the result of accident, and their successful treatment. The indications for operation depend on the severity of the symptoms and on the presence of either haemorrhage or inflammation, which may demand operation. If, therefore, after an injury in the superior abdominal region, there be increasing anaemia, the signs of free blood in the peritoneum, or peritonitis, the surgeon will be called on to operate without delay.

Severe injuries of the pancreas not operated on terminate fatally in nearly every case. Out of 45 cases of pancreatic injury collected by von Mikulicz, 21 were perforating, 12 were gunshot, and 9 were stab wounds. Of the gunshot injuries 5 were operated on, 3 recovering; the 7 not operated on all died. Of the stab wounds all were operated on, and 8 out of 9 recovered. Of the 24 subcutaneous injuries 13 were not operated on and all died. Of the 11 operated on 7 recovered.

No hard-and-fast rule can be formulated as to the method of treatment; the injured part must be exposed and either ligature of vessels or suture of surfaces adopted; but gauze tampons and drainage form important features in any of these operations, and where the peritoneum has been generally soiled with blood and pancreatic effusion, lavage with hot saline fluid affords the double advantage of relieving shock and cleansing the abdomen.

In view of the favourable results obtained by operation and the lethal effects of non-operative treatment, the only lesson that can be drawn is that we should make an exploratory laparotomy whenever there is a question of severe pancreatic injury. 